Document downloaded from:

http://hdl.handle.net/10251/69265

This paper must be cited as:

Santiago Felipe, S.; Tortajada-Genaro, LA.; Carrascosa Rubio, J.; Puchades, R.; Maquieira Catala, Á. (2016). Real-time loop-mediated isothermal DNA amplification in compact disc micro-reactors. Biosensors and Bioelectronics. 79:300-306. doi:10.1016/j.bios.2015.12.045.

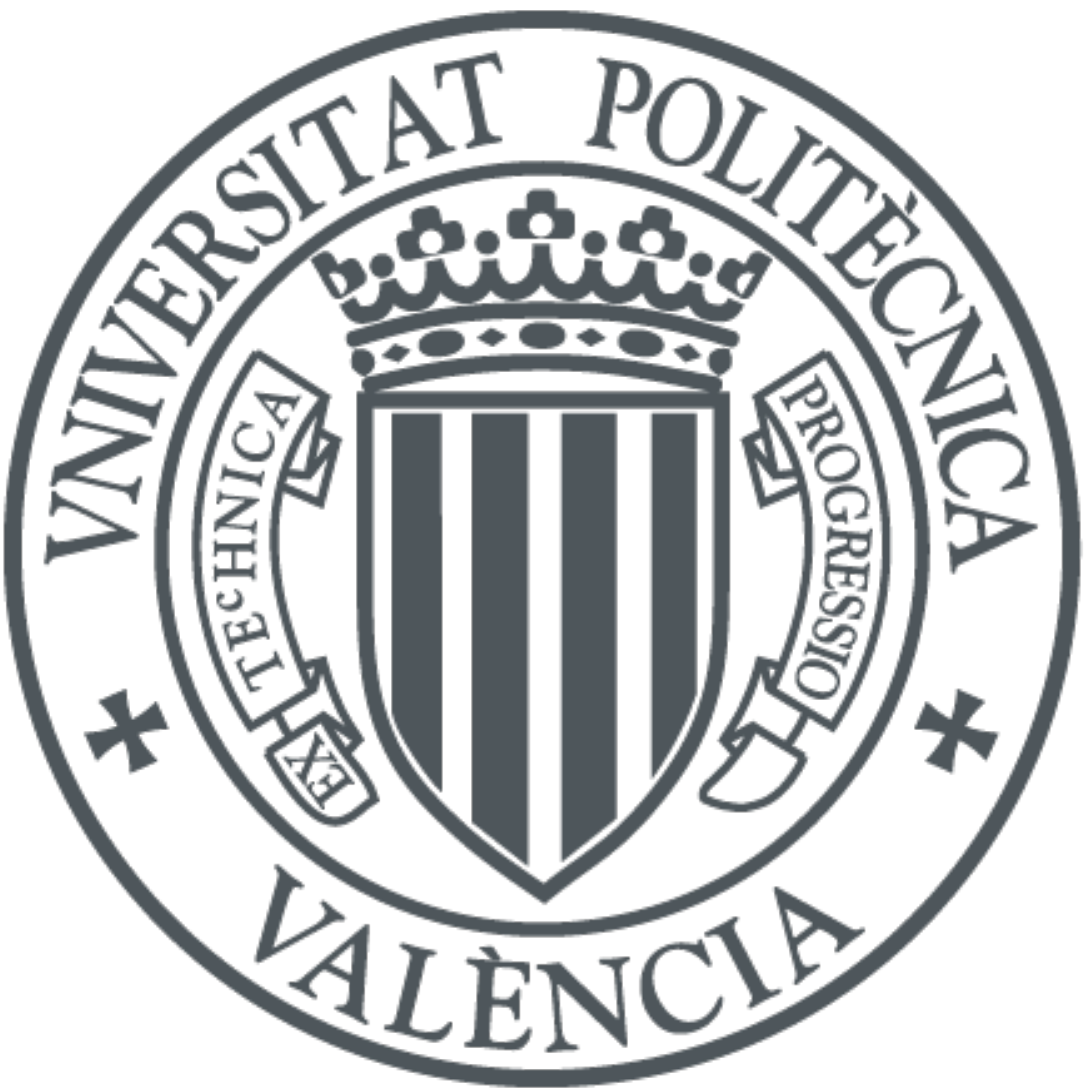

The final publication is available at

https://dx.doi.org/10.1016/j.bios.2015.12.045

Copyright Elsevier

Additional Information 


\title{
Real-time loop-mediated isothermal DNA amplification in compact disc micro-reactors.
}

Sara Santiago-Felipe, Luis A. Tortajada-Genaro, Javier Carrascosa, Rosa Puchades, 5 Ángel Maquieira

Instituto Interuniversitario de Reconocimiento Molecular y Desarrollo Tecnológico (IDM), Departamento de Química, Universitat Politècnica de València, Camino de Vera s/n, E46071 Valencia, Spain

\begin{abstract}
An integrated device composed of micro-reactors embedded onto compact discs is proposed for real-time targeted DNA determination. The method principle is based on in-disc loop-mediated isothermal amplification (iD-LAMP) and quantitative optical

15 read-out by a disc drive. In the presence of a target, the turbidimetric or colorimetric properties of reaction solution change, and the transmitted intensity of the disc drive laser modifies according to reaction yield. Monitoring real-time curves allowed the quantitative determination of DNA template amounts. The best amplification/detection results were obtained with micro-reactors $(2 \mathrm{~mm}$ diameter and $1.1 \mathrm{~mm}$ in depth) drilled on a digital video disc (DVD) and detection based on the colorimetric mode. As proofof-concept, the assay was applied to detect pathogenic bacteria Salmonella spp. and to identify bovine meat in food samples. Ninety-six samples were simultaneously analysed in $15 \mathrm{~min}$, with high selectivity and sensitivity $(5 \mathrm{CFU} / \mathrm{mL}$ and $10 \mu \mathrm{g} / \mathrm{g}$ for bacteria and meat, respectively). The in-disc results were comparable to those obtained by conventional LAMP or qPCR approaches. The developed device allows low sample and reagent consumption ( $3 \mu \mathrm{L}$ of reaction), portability, ease-of-use, and rapid low-cost high-throughput analyses.
\end{abstract}

Keywords: DNA amplification, LAMP, Isothermal real-time, Micro-reactors, Compact disc. 


\section{Introduction}

Nucleic acid-based methods are preferred for analytical detection in several areas, such as food safety, environmental monitoring and clinical diagnostics. A key step in DNA detection is selective amplification because it generates a large number of target copies, which vastly increases assay sensitivity and controls selectivity (Asiello and Baeumner, 2011). The most widespread method for amplification is polymerase chain reaction (PCR) performed in benchtop thermocyclers. However, efforts are being made to develop inexpensive, user-friendly small-sized devices for point-of-care applications (McCalla and Tripathi, 2011; Marx, 2015). Miniaturised and microfluidic reactors match these goals as they cut reaction times, consumption of samples and reagents, and increase portability and the automation potential (Auroux et al., 2004; Zanoli and Spoto, 2013).

Although different PCR-based devices have been developed, some limitations hinder them bring integrated into biosensing systems (Focke et al., 2010). PCR demands an accurate temperature control and rapid thermocycling at between $55^{\circ} \mathrm{C}$ and $95^{\circ} \mathrm{C}$. The fluctuation of temperature when initiating a specific step results in overshooting or undershooting (Asiello and Baeumner, 2011). Besides, high temperatures lead to variations in the volume reaction and gas bubble formation, one of the main drawbacks in lab-on-a-chip. These constraints can be overcome through isothermal amplification methods, where nucleic acids are synthesised at constant temperature using proteins that separate DNA strands (Craw and Balachandran, 2012). In isothermal amplification, the reaction time is controlled by enzyme activity rather than the rate of thermal cycling and heat transport, as occurs in PCR-based methods. This offers the advantage of simple design combined with less energy consumption, which makes amplification compatible with portable detection systems.

Among the developed isothermal techniques, loop-mediated isothermal amplification (LAMP) has become a notable option thanks to its performance. LAMP uses Bst DNA polymerase to create loop structures that facilitate exponential sequencespecific amplification (Hara-Kudo et al., 2005). Under isothermal conditions $\left(60-65^{\circ} \mathrm{C}\right)$, this method generates large amounts of product ( $>10^{9}$ amplification factor) within $1 \mathrm{~h}$. The use of multiple target sequence regions (4-6 primers) confers high specificity. Therefore, LAMP is a very sensitive, easy, and time-saving method that is being successfully applied in a phial format (Chen et al., 2011; Tanner et al., 2012). Some 
devices based on end-point detection or real-time monitoring of LAMP reactions coupled to electrochemical detection (Hsieh et al., 2012; Safavieh et al., 2014), absorbance detection (Fang et al., 2010), fluorescence detectors (Zhou et al., 2014) or charge-coupled device-based fluorescence imaging systems (Ahmad et al., 2011) have been described. However, some of these approaches are simply not practical or expensive.

Genomic assays are also performed in centrifugal devices, which integrate all the analytical steps by controlling the rotation rate (Mori et al., 2004) or involving some handling steps (Roy et al., 2015). These "lab-on-a-disc" systems generally consist of microstructured circular platforms (channels, valves, chambers, etc.) where the reaction takes place, and the measurement is taken with standard instruments, such as colorimeters, or with expensive static detectors, such as fluorescence microscopes, scanners or other complex non-integrated systems. In this study, a novel method called

80 in-disc LAMP (iD-LAMP) was developed by integrating LAMP amplification and compact disc technology. Our approach is based on the direct use of standard audiovideo discs (CD, DVD) as a biosensing platform to carry out DNA amplification, and to read results by optical scanning with the disc drive laser. Compared to DNA biosensors based on detection by a disc drive (Morais et al. 2009; Lutz et al., 2010; TortajadaGenaro et al., 2012; Tortajada-Genaro et al., 2015), DNA amplification reactions are performed in-disc and progress is real-time monitored.

\section{Materials and methods}

\subsection{Discs}

The amplification platform comprised three layers (Figure 1a). The first was a modified optical disc: compact disc (CD), digital versatile disc (DVD) or Blu-Ray discs (BD). The Supplementary Material includes the protocols used for the fabrication of micro-reactors and for the oxygen plasma activation of discs. The second layer consisted of a double-sided pressure-sensitive adhesive of $0.08 \mathrm{~mm}$ in depth (ARcare 90445, Adhesives Research, Ireland). Chambers of $5 \times 4.5 \mathrm{~mm}$ were cut using a cutterplotter (Graphtec, Japan-Graphtec CE-2000). Once the disc and adhesive layer had been machined, they were aligned and laminated together. An acetate sheet composed the 
third layer, used to seal the system. The resulting disc contained micro-reactors for 96 assays.

\subsection{Detector}

A detailed description of readers, manufactured from standard disc drives, is included in the Supplementary Material. The measurement principle was based on the variation of the optical disc properties due to the presence of amplification products in the micro-reactor (Figure 1b). The detector measured the changes in the light intensity

105 of the drive laser transmitted through solutions during a cyclic scanning of the surface (disc rotation).

The device was enabled and configured to the specified disc rotational speed $(1,000 \mathrm{rpm})$, laser power $(1 \mathrm{~mW})$, and laser beam focus. Cyclic scanning of the disc area that contained micro-reactors $(35-45 \mathrm{~mm}$ from the disc centre) was performed.

110 During each cycle (duration: $2 \mathrm{~min}$ ), automatic laser power tuning was carried out, and the power drift due to temperature conditions $\left(60^{\circ} \mathrm{C}\right)$ and intrinsic variations to the laser temperature was corrected. The transmitted optical density of the drive laser was recorded at an acquisition signal of $26 \mathrm{~dB}$ gain and 1 mega-samples per second. The data stream was divided into peaks that correlated with the micro-reactors of the disc,

115 synchronized at the same start point through a footprint on the outer disc edge. The averaged peaks (ten readings per cycle) were recorded in a character-separated values file (CSV). The optical signal was monitored during amplification, and reaction progress was recorded.

\subsection{Samples}

The sequences of the LAMP primers for detecting Salmonella spp. and bovine species are shown in Table S1. Reference bacterial strains (targeted and non-targeted serovars and organisms) were supplied by CECC (Spain), NCTC (UK) and ATCC (USA). Bacterial isolation and inoculation assays were prepared as described by Santiago-Felipe et al., 2014. Meat products were bought in local stores. Genomic DNA was extracted by the DNeasy Blood \& Tissue Kit (Qiagen, Inc., USA). 


\subsection{In-disc LAMP reaction and detection}

Amplification reactions were carried out in $3-\mu \mathrm{L}$ micro-reactors. The reaction mixture contained 1× ThermoPol reaction buffer (New England BioLabs, USA), $6 \mathrm{mM}$

$\mathrm{MgSO}_{4}, 1.2 \mathrm{mM}$ dNTPs, $0.2 \mu \mathrm{M}$ F3 and B3, $1.6 \mu \mathrm{M}$ FIP and BIP, $0.64 \mathrm{M}$ betaine, $16 \mathrm{U}$ of Bst DNA polymerase (New England BioLabs), $2.5 \mathrm{ng}$ of DNA template, and inert oil $(8 \%, v / v$, Sigma) to prevent evaporation. For the Salmonella spp. amplification, the mixture also included $0.8 \mu \mathrm{M}$ of primers LF and LB. Depending on the detection strategy, other reagents were added: no reagent for direct turbidimetry, $1 \mu \mathrm{M}$

135 polyethylenimine (PEI) at the end of the reaction for end-point turbidimetry, and 120 $\mu \mathrm{M}$ hydroxynaphthol blue (HNB) in the reaction mixture for colorimetry.

Micro-reactors were sealed (acetate sheet) and inserted into the disc drive. The reader was placed in an oven (Memmert, model UF30, Germany) at $65^{\circ} \mathrm{C}$. The disc was cyclically scanned during isothermal incubation (duration: $30 \mathrm{~min}$, cycle frequency: 2

$140 \mathrm{~min}^{-1}$ ). A sample was considered positive when the optical response was greater than the cut-off value (threshold), calculated as 3 times the standard deviation of the negative control. The concentration was determined from the threshold time, the time needed for each sample to exceed the quantification signal, measured for both samples and standards. Used discs were discarded following the same laboratory safety guidelines as

145 ELISA plates.

\subsection{Complementary/Secondary measurements}

The Supplementary Material describes the measurement of the micro-reactor dimensions, contact angle values, and particle size distribution of the particles produced during the reactions. For a comparison with real-time measurements by the benchtop

150 instrument, $1 \mu \mathrm{L}$ of 1:10 diluted original SYBR green dye I (Invitrogen, Spain) was added to the in-tube LAMP reactions (96-well plate and total volume of $25 \mu \mathrm{L}$ ) before incubation at $65^{\circ} \mathrm{C}$ in a 7500 Real-Time System (Applied Biosystems, Spain). Fluorescence readings were obtained every $2 \mathrm{~min}$ and the results were considered positive when the fluorescence signal exceeded a threshold value (40,000 units).

155 Statistical package Statgraphics Centurion for Windows v. 16.1.15 was used for the data analysis. 


\section{Results}

\subsection{Micro-reactors design}

Three optical disc technologies (CD, DVD, and BD) were tested to carry out the iD-LAMP method. Firstly, the compatibility of laser reading with the fabrication of a micro-reactor inside the disc was studied. In particular, the dimensions of the microreactors could alter the physical disc properties and the capability to follow the data track, present in the grove polycarbonate. A two-factor experimental design was performed by varying the diameter $(0.8$ to $4 \mathrm{~mm})$ and depth $(0.6$ to $1.1 \mathrm{~mm})$ of the embedded micro-reactors. With this configuration, the reflective metallic layer was locally removed, which allowed light to be transmitted through specific structured disc areas. Although disc integrity did not change, none of the structured BD discs was recognized by the $\mathrm{BD}$ drive. With CDs or DVDs, micro-reactors with diameters shorter than $2 \mathrm{~mm}$ were compatible with the reading, which allowed full disc surface scanning.

The differences observed between optical reading technologies were associated with error tolerance, particularly burst errors. Disc drives include a correction system to minimize defects on disc surfaces like scratches, dust, fingerprints, etc. Given its minor laser spotting Blu-Ray technology, the thin cover layer and the high numerical aperture were more sensitive to burst errors than the CD and DVD ones. For the biosensing application, the micro-reactors in the disc were like repetitive burst errors. Therefore, when the laser focused on a given in-disc micro-reactor, disc scanning followed if the disc drive was capable of correcting that error. In conclusion, BDs were less tolerant to defects than CDs or DVDs and were, therefore, less robust to cope with microstructures in the disc.

180 The following experiments focused on investigating the sensing capabilities based on detecting the light transmitted at a fixed time or during a given time interval (see Supplementary Material). The results showed that the response heavily depended on the micro-reactor depth due to optical pathway variation. The selected dimensions were 2 $\mathrm{mm}$ diameter and $1.1 \mathrm{~mm}$ in depth. Besides, kinetic measurements were also possible using the proposed device because the detector was able to capture the signal in the indisc micro-reactors by cyclic surface scanning. Therefore, both sets of experiments demonstrated that the micro-reactors embedded in CDs and DVDs did not disturb track 
scanning, which facilitates the measurement of reaction solutions (end-point or realtime) by disc drives.

\subsection{Disc treatment}

The hydrophobic nature of the disc substrate (polycarbonate, contact angle $\sim 90^{\circ}$ ) may hinder the dispensing of reagents inside in-disc micro-reactors and may lead to additional problems, such as non-specific adhesion to micro-reactor walls. As previously demonstrated for microfluidic channels (Liu et al., 2001; Mori et al. 2006), ${ }^{\text {the }}$ micro-reactor surface was hydrophilised to overcome these drawbacks.

In a first approach, liquid treatments were assayed and contact angles were measured (Table 1). Structures were treated with surfactant Hellmanex II (120 min at room temperature with a $2 \%$ solution in demineralised water), acid treatment $(20 \%$

$200 \mathrm{HNO}_{3}+5 \% \mathrm{H}_{2} \mathrm{SO}_{4}$ for $10 \mathrm{~min}$ at room temperature), and alkaline treatments $(\mathrm{NaOH}$ $1 \mathrm{M}$ for $30 \mathrm{~min}$ at $60^{\circ} \mathrm{C}$ ). These methods only reduced hydrophobicity by $12 \%$. Then, physical treatments (UV irradiation and plasma exposure) were studied. Exposure to UV-light with a mercury capillary lamp $\left(6 \mathrm{~mW} / \mathrm{cm}^{2}\right)$ at two different wavelengths $(371$ and $254 \mathrm{~nm}$ ) for $240 \mathrm{~min}$ significantly decreased the contact angle (Table 1). This reduction was greater for the shorter wavelength, with reduction percentages of $55 \%$ and $81 \%$ for $\lambda=371 \mathrm{~nm}$ and $\lambda=254 \mathrm{~nm}$, respectively. For oxygen plasma activation, discs were introduced into a plasma system which operated at $2.45 \mathrm{GHz}, 100 \mathrm{~W}$ and $120 \mathrm{~Pa}$ for $30 \mathrm{~s}$ and a hydrophilic surface was achieved with a $20^{\circ}$ contact angle (Table 1). After disc treatment, reagent solutions were easily loaded in the in-disc micro-

210 reactor and no surface interaction was observed. This made it the fastest, cleanest, easygoing, and most effective method. Under these conditions, surface topography was not modified, optical properties remained unchanged, the ageing effect was minimum, and the surface was hydrophilic after 1-month storage.

\subsection{Characterisation of iD-LAMP products}

215 As proof-of-concept, the detection of a pathogenic bacterium (Salmonella spp.) and the identification of meat species (bovine) were studied. The reactions were performed in-disc (reaction volume: $3 \mu \mathrm{L}$ ) and in polypropylene tubes (reaction volume: $25 \mu \mathrm{L})$. The ladder-like patterns of gel electrophoretic separation and amplification 
yields were comparable for both amplification products (Supplementary Material). No

220 small fragments were observed for the slow heat transfer described in some amplification devices (Lee et al., 2008). Effective in-disc elongation by Bst DNA polymerase was achieved because the disc substrate (polycarbonate) displayed excellent thermal conductance (thermal conductivity of $0.241 \mathrm{~W} \cdot \mathrm{m}^{-1} \cdot \mathrm{K}^{-1}$ and thermal diffusivity constant of $1.5310^{-7} \mathrm{~m}^{2} \cdot \mathrm{s}^{-1}$ at $\left.38^{\circ} \mathrm{C}\right)$.

\subsection{Detection strategies of iD-LAMP products}

Several strategies were compared to quantify the products generated in the in-disc micro-reactors. Turbidimetric sensing focused on magnesium pyrophosphate, a byproduct of DNA amplification, or combined with the insoluble complex between synthetised DNA products and polyethylenimine (PEI) (Mori et al., 2006). The second tested strategy was the colorimetric detection mode based on adding hydroxynaphthol blue (HNB) to the initial LAMP reaction mixture (Goto et al., 2009).

The initial experiments focused on measuring the optical properties of the products formed in-disc. For this purpose, solutions were recovered from micro-reactors

235 after amplification and were analysed by common optical techniques (dispersion and absorption). The first approach involved taking turbidity measurements by the formation of magnesium pyrophosphate or the DNA-PEI complex. Effective beam dispersion measurement required particle sizes to be above $\lambda / 4$, where $\lambda$ was the disc driver laser wavelength (CD $\lambda=780 \mathrm{~nm}$ and DVD $\lambda=650 \mathrm{~nm})$. The measurement of the

240 size distribution profiles indicated that in-disc amplification produced a suspension that contained submicro-particles with a mean diameter of $336 \pm 119 \mathrm{~nm}$ (Supplementary Material). The study of submicro-particles formed by DNA-PEI interaction indicated that the proportion of high-diameter particulates was higher (mean diameter $660 \pm 261$ $\mathrm{nm}$ ). The visible spectra of amplified products were recorded between 500 and $800 \mathrm{~nm}$

245 (Figure 2a). In both cases, a nearly constant response was obtained for the selected wavelength interval. The widest variation of the optical signal was achieved after the end-point addition of PEI within the $0.5-10 \mu \mathrm{M}$ range.

Regarding the second tested strategy, $\mathrm{MgP}_{2} \mathrm{O}_{7}$ formation as a result of DNA amplification produced the red/purple Mg-HNB complex dissociation, so the HNB 250 returned to its uncomplexed form, blue under the buffered reaction conditions ( $\mathrm{pH} 8$ ). 
The visible spectrum (Figure 2a) showed an overlap between the reduction of the transmitted light associated with $\mathrm{MgP}_{2} \mathrm{O}_{7}$ precipitate formation and an absorption peak associated with blue Mg-HNB complex formation (Figure 2a). The wavelength of the peak maximum coincided with that of the DVD driver $(650 \mathrm{~nm})$.

In-disc detection by the tested devices (CD and DVD readers) in the transmission mode was studied (Figure $2 b$ ). The worse results were obtained by direct turbidity since only the largest DNA template amounts (up to $10 \mathrm{pg} / \mu \mathrm{L}$ ) produced significant laser beam disruption. The addition of PEI $(1 \mu \mathrm{M})$ led to a major signal variation for both readers. The addition of HNB $(120 \mu \mathrm{M})$ resulted in higher and lower responses using a

260 DVD drive and a CD drive, respectively. Therefore, the results indicated that both the detection strategies, turbidity (DNA-PEI complex/both readers) and colorimetric (magnesium-HNB complex/DVD reader), can be suitable for the detection of iD-LAMP products in sensitivity and reproducibility terms. However, the post-amplification addition of PEI increased the risk of contamination and difficulties for automation

265 and/or integration processes. The initial addition of HNB to the reaction mixture was selected as the best detection strategy using a DVD platform.

An initial approach was the end-point determination of targeted DNA regions (Supplementary Material). Figure 2c shows the optical responses measured by the DVD drive after a 30-minute reaction according to the DNA template concentration. These

270 experiments proved that the colour changes detected by the DVD drive reflected the amplification yield while the reaction progressed. The lowest detected concentrations were $65 \mathrm{fg} \mathrm{DNA} / \mu \mathrm{L}$ (equivalent to $13 \mathrm{CFU} / \mathrm{mL}$ ) and $0.3 \mathrm{fg} \mathrm{DNA} / \mu \mathrm{L}$ (equivalent to the $9 \mu \mathrm{g} / \mathrm{g}$ sample) for Salmonella spp. and bovine genes, respectively.

\subsection{Real-time detection}

To monitor the iD-LAMP amplification kinetics, genomic DNA was amplified indisc by recording colour changes (cycle: $2 \mathrm{~min}$ ). The same samples were also amplified in-tube in a conventional thermal cycler and were monitored by a fluorescence marker. The signal-time registers obtained for both methods are shown in Figure 3a and $3 \mathrm{~b}$.

280 Although the final amplification yields were comparable, the in-disc assay gave a higher amplification rate than the in-vial one. Probably, the enlarged surface-to-volume ratio increased thermal transfer and thus reduced cycling times, as demonstrated in other miniaturised devices (Lee et al., 2008). An increase in the quantity of the initial 
template DNA reduced the threshold time, or the reaction time need to reach positive signals above the baseline (optical density 0.07 for the DVD drive and 40,000 units for a conventional thermal cycler).

The calibration plot (threshold time vs. the logarithm of the initial DNA template concentration) showed a linear relationship for both Salmonella spp. and bovine systems $\left(r^{2}=0.987\right.$ and $r^{2}=0.985$, respectively), (Figures $3 c$ and $\left.3 d\right)$. The limits of

290 detection were $5 \mathrm{CFU} / \mathrm{mL}$ and $10 \mu \mathrm{g} / \mathrm{g}$ sample for Salmonella spp. and bovine, respectively. The relative standard deviation in five replicates was $6.5-17.1 \%$. These results revealed that the iD-LAMP method showed adequate sensitivity, a good working range, and sound reproducibility for the quantitative DNA analysis in under $15 \mathrm{~min}$.

The reliability of the iD-LAMP method to be applied to large-scale screening was evaluated by analysing different pathogen cultures and foodstuffs, and by comparing it with a reference method (qPCR). Specific genes for Salmonella spp. (invA gene) and bovine species (mitochondrial 12S rRNA gene) were analysed. Table 2 summarises the results of the iD-LAMP (96-micro-reactors-DVD) and qPCR methods. The iD-LAMP concentrations were in good agreement with the qPCR concentrations for both analytes (paired t-test, p-values: 0.086 and 0.760 , respectively).

\subsection{Comparison to other approaches}

Numerous partially or totally automated methods have been described for DNA assays. They include PCR in "lab-on-a-chip" devices (McCalla and Tripathi, 2011), PCR in disc-shape approaches (Gorkin et al., 2010; Roy et al., 2015), LAMP in 305 conventional plates (Tanner et al. 2012; Denschlag et al., 2013), LAMP in integrated devices (Ahmad et al., 2010; Hsieh et al., 2012; Safavieh et al., 2014; Zhou et al., 2014) and other isothermal approaches (Santiago-Felipe et al.; 2014; Tortajada-Genaro et al., 2015). These techniques have reported similar and, in a few cases, better analytical performance (i.e. limit of detection 10-1000 fg/ $\mu \mathrm{L}$ ) than iD-LAMP, but have some

310 drawbacks. In some cases, the results are obtained at the end-point, or fewer samples can be analyzed simultaneously. Some approaches need complex detection equipment which considerably increases and limits their costs and portability.

The use of an iD-LAMP biosensor offers interesting advantages for end-point and real-time analyses. Firstly, our device takes advantage of intrinsic disc photonics

315 (semiconductor diode, laser positioning/focusing system, etc.). It is worth mentioning that using a high-intensity DVD laser beam, instead of other approaches such as a 
source of monochromatic light at $650 \mathrm{~nm}$ and a camera, leads to a more compact instrument, which yields high quality images. Furthermore, the mass production of discs and readers assures a high quality, low-cost and high-access sensors. Optical reader properties (laser drive), such as lightweight ( $<500 \mathrm{~g}$ ) or small volume (a few $\mathrm{cm})$, make them competitive to be implemented into different settings. The prospective costs of the detection system $(<€ 3 /$ disc and $<€ 300 /$ reader $)$ are below the state-of-art, i.e. qPCR plates and fluorescence-thermal cyclers. Secondly, polycarbonate substrates, treated by oxygen plasma, offer excellent bioanalytical properties compared to other plastics, such

325 as stability, minimal non-specific adsorption and low signal backgrounds. Thirdly, as the design is based on an isothermal amplification technique, the method does not require fast accurate temperature changes, expensive reagents or time-consuming steps. iD-LAMP has demonstrated high specificity amplification in short reaction times. Furthermore, the technician "hands-on" time for the iD-LAMP assay is estimated to be 330 one third that of a manual qPCR test. Fourthly, the working capability for real-time monitoring is greater than conventional in-vial or alternative cartridge approaches. As proof-of-concept, the assayed device has 96 micro-reactors, but the number of samples simultaneously analysed can increase given the dimensions of discs (surface area of 94 $\mathrm{cm}^{2}$ ) and micro-reactors (surface area of $3.1 \mathrm{~mm}^{2}$ ).

\section{4. Conclusions}

The iD-LAMP system is a real-time non-fluorescent development that meets the requirements of DNA-based biosensing such as effective, low-cost and fast response. The research efforts made in this study have focused on testing the features of LAMP methods combined with optical disc technology owing to its simplicity and robustness.

340 The integration of micro-reactors, by simply drilling the plastic substrate onto optical discs, and the later sensing of the light transmitted by the disc drive, have been demonstrated. The detection of targeted DNA based on an economically affordable analytical system is achieved. Nevertheless, a heating system should be integrated with the DVD-based device for out-of-lab use. The system can be equipped with different components to monitor and control enclosure temperatures. An effective solution could include a Peltier heater, embedded thermocouples and a proportional-integral-derivative temperature controller. In any case, simple cheap accessories can be implemented into the reader, which are compatible with all the other components. 
The method feasibility has been assessed by determining two different kinds of 350 analytes, such as pathogen detection and species identification. iD-LAMP technology has shown excellent analytical performance (selectivity, sensitivity, reproducibility, and high throughput), and the results are comparable to in-vial LAMP and qPCR. This advantageous DNA quantification method is suitable for being applied in a wide range of areas, such as low resource settings, satellite/decentralised laboratories and 355 production plants.

\section{Acknowledgements}

This research has been funded through projects FEDER, CTQ/2013/45875-R (MINECO) and PrometeoII/2014/040 (GVA). The Spanish Ministry of Education and Science provided S.S.F. with a grant for her $\mathrm{PhD}$ studies.

\section{References}

Ahmad, F., Seyrig, G., Tourlousse, D.M., Stedtfeld, R.D., Tiedje, J.M., Hashsham, S.A., 2011. Biomed. Microdevices 13 (5), 929-937.

Asiello, P.J., Baeumner, A.J., 2011. Lab Chip 11 (8), 1420-1430.

365 Auroux, P.A., Koc, Y. deMello, A. Manz, A. Day, P.J.R. 2004. Lab Chip 4 (6), 534546.

Chen, L., Guo, J., Wang, Q., Kai, G., Yang, L.J., 2011. Agric. Food Chem. 59 (11), 5914-5918.

Craw, P., Balachandran, W., 2012. Lab Chip 12 (14), 2469-2486.

370 Denschlag, C., Vogel, R.F., Niessen, L., 2013. Int. J. Food Microbiol. 162 (3), 245-251.

Fang, X., Liu, Y., Kong, J., Jiang, X., 2010. Anal. Chem. 82 (7), 3002-3006.

Focke, M., Stumpf, F., Roth, G., Zengerle, R., von Stetten, F., 2010. Lab Chip 10 (23), 3210-3212.

Gorkin, R., Park, J., Siegrist, J., Amasia, M., Lee, B.S., Park, J., Kim, J., Kim, H., Madou, M., Kyoung Cho, Y., 2010. Lab Chip 10 (14), 1758-1773.

Goto, M., Honda, E., Ogura, A., Nomoto, A., Hanaki, K., 2009. BioTechniques 46, $167-$ 172.

Hara-Kudo, Y., Yoshino, M., Kojima, T., Ikedo, M., 2005. FEMS Microbiol. Lett. 253 (1), 155-161. 
380 Hsieh, K., Patterson, A.S., Ferguson, B.S., Plaxco, K.W., Soh, H.T., 2012. Angew. Chem. Int. Ed. 51, 4896-4900.

Lee, S., Huang, J., Chuang, T., Sheu, J., Chuang, Y., Holl, M., Meldrum, D.R., Lee, C., Lin, C., 2008. Sensor. Actuat. B-Chem. 133, 493-501.

Liu, Y., Ganser, D., Schneider, A., Liu, R., Grodzinski, P., Kroutchinina, N., 2001. 385 Anal. Chem. 73, 4196-4201.

Lutz, S., Weber, P., Focke, M., Faltin, B., Hoffmann, J., Müller, C., Mark, D., Roth, G., Munday, P., Armes, N., Piepenburg, O., Zengerle, R., von Stetten, F., 2010. Lab Chip 10, 887-893.

Marx, V. 2015 Nat. Methods 12, 393-397

390 McCalla, S.E., Tripathi, A., 2011. Annu. Rev. Biomed. Eng. 13, 321-343.

Morais, S., Tortajada-Genaro, L.A., Arnandis-Chover, T., Puchades, R., Maquieira, A., 2009. Anal. Chem. 81, 5646-5654.

Mori, Y., Hirano, T., Notomi, T., 2006. BMC Biotechnol. 6, 3-12.

Mori, Y., Kitao, M., Tomita, N., Notomi, T.J., 2004. Biochem. Bioph. Meth. 59, 145395 157.

Roy, E., Stewart, G., Mounier, M., Malic, L., Peytavi, R., Clime, L., Madou, M., Bossinot, M., Bergeron, M.G., Veres, T., 2015. Lab Chip 15, 406-416.

Safavieh, M., Ahmed, M.U., Ng, A., Zourob, M., 2014. Biosens. Bioelectron. 58, 101106.

400 Santiago-Felipe, S., Tortajada-Genaro, L.A., Morais, S., Puchades, R. Maquieira, A., 2014. Sensor. Actuat. B-Chem. 204, 273-281.

Santiago-Felipe, S., Tortajada-Genaro, L.A., Puchades, R., Maquieira, A., 2014. Anal. Chim. Acta 811, 81-87.

Tanner, N.A., Zhang, Y., Evans, T.C., 2012. BioTechniques 53, 81-89.

405 Tortajada-Genaro, L.A., Santiago-Felipe, S., Amasia, M., Russom, A., Maquieira, A., 2015. RSC Adv. 5, 29987-29995.

Tortajada-Genaro, L.A., Santiago-Felipe, S., Morais, S., Gabaldón, J.A., Puchades, R., Maquieira, A., 2012. J. Agr. Food Chem. 60, 36-43.

Zanoli, L.M. Spoto, G. 2013. Biosensors 3, 18-43.

410 Zhou, Q., Wang, L., Chen, J., Wang, R., Shi, Y., Li, C., Zhang, D., Yan, X., Zhang, Y.J., 2014. Microbiol. Meth. 104, 26-35. 


\section{LIST OF FIGURES}

415 Figure 1. (a) Photography of the micro-reactor: bottom layer: modified DVD disc; Intermediate layer: two-sided pressure-sensitive adhesive; Upper layer: acetate sheet. (b) Photograph of micro-reactors embedded on a DVD disc and schematic diagram of the detection system by a DVD drive.

420 Figure 2. Results obtained by the tested detection methods: turbidity based on magnesium pyrophosphate precipitate (direct), turbidity based on the DNA-PEI complex (PEI), and colorimetry based on Mg-HNB complex formation (HNB). (a) Absorption spectra of iD-LAMP products. Colour dashed lines indicate the wavelength of each disc drive: Digital Versatile Disc (DVD) and Compact Disc (CD). (b)

425 Maximum signals obtained by CD and DVD drives (DNA concentration $50 \mathrm{pg} / \mu \mathrm{L}$ ). (c) Signals obtained by a DVD drive based on Mg-HNB complex formation for different DNA concentrations (extracted DNA from Salmonella culture).

Figure 3. (a) In-disc LAMP response monitored via colorimetric detection by the DVD 430 reader (b) In-tube LAMP response monitored via fluorescence using an Applied Biosystem 7500 thermal cycler. (c). Relation between threshold times and the logarithm of the amount of the DNA template for Salmonella spp. (d) Relation between threshold times and the logarithm of the DNA template amount for bovine. Five replicates. 


\section{LIST OF TABLES}

Table 1. Contact angle measurements and reduction percentage according to the surface treatment. Replicates: 5 droplets.

440

Table 2. Quantitative results for the samples analysed by iD-LAMP and qPCR as a reference method: (A) Salmonella spp. and (B) bovine. Five replicates. 